\title{
Deformation-Induced Microstructural Banding in TRIP Steels
}

\author{
S. CELOTTO, H. GHADBEIGI, C. PINNA, B.A. SHOLLOCK, and P. EFTHYMIADIS
}

\begin{abstract}
Microstructure inhomogeneities can strongly influence the mechanical properties of advanced high-strength steels in a detrimental manner. This study of a transformation-induced plasticity (TRIP) steel investigates the effect of pre-existing contiguous grain boundary networks (CGBNs) of hard second-phases and shows how these develop into bands during tensile testing using in situ observations in conjunction with digital image correlation (DIC). The bands form by the lateral contraction of the soft ferrite matrix, which rotates and displaces the CGBNs of second-phases and the individual features within them to become aligned with the loading direction. The more extensive pre-existing CGBNs that were before the deformation already aligned with the loading direction are the most critical microstructural feature for damage initiation and propagation. They induce micro-void formation between the hard second-phases along them, which coalesce and develop into long macroscopic fissures. The hard phases, retained austenite and martensite, were not differentiated as it was found that the individual phases do not play a role in the formation of these bands. It is suggested that minimizing the presence of CGBNs of hard second-phases in the initial microstructure will increase the formability.
\end{abstract}

https://doi.org/10.1007/s11661-018-4650-z

(C) The Author(s) 2018

\section{INTRODUCTION}

TRANSFORMATION-INDUCED plasticity (TRIP) steels are one class of advanced high-strength steels (AHSS) that have been developed for applications in the automotive industry in recent years. ${ }^{[1,2]}$ Their combination of high strength and increased uniform and total elongation, even compared to dual-phase (DP) steels, enables the forming of complex-shaped components where stretchability is a key formability characteristic. Additionally, this combination of properties can enhance the energy absorption capacity, giving better crashworthiness and thus, improved passenger safety. However, this new class of material exhibits microstructural banding, a common detrimental microstructural feature found in many steel types. These bands in general consist of alloying addition (e.g., $\mathrm{C}, \mathrm{Mn}$ ) enriched microstructural constituents, usually pearlite or martensite, as layers parallel to the rolling plane.

S. CELOTTO is with Tata Steel R\&D, IJmuiden, 1970 CA, The Netherlands. H. GHADBEIGI and C. PINNA are with the Department of Mechanical Engineering, University of Sheffield, Mappin Street, Sheffield, S1 3JD, UK. B.A. SHOLLOCK and P. EFTHYMIADIS are with the WMG, University of Warwick, Coventry CV4 7AL, UK. Contact e-mail: P.Efthymiadis@warwick. ac.uk

Manuscript submitted December 18, 2017.

Article published online May 11, 2018
They form as a consequence of the processing at both casting and rolling deformation stages ${ }^{[3]}$ This study, like others, shows that these bands have an important effect on mechanical properties. ${ }^{[4-6]}$

There have been previous studies to investigate and control the formation of microstructural bands during processing. ${ }^{[7,8]}$ It has been reported that the thermomechanical processing required to avoid the formation of microstructural bands can be sometimes economically impractical ${ }^{[7]}$ or physically impossible due to the thermodynamics and kinetics governing the formation of such bands. ${ }^{[8]}$ Nevertheless, a better understanding of the effects of such bands on the microstructural behavior of TRIP steels can lead to the development of new processing routes aimed at improving the mechanical properties of these materials by optimizing the geometric characteristics of these bands. ${ }^{[3,9]}$ Furthermore, some optimizations can be realized through economically feasible thermomechanical processes ${ }^{[3,9]}$ For example, it has been suggested that increasing the cooling rate during the hot rolling process leads to a significant decrease of martensite banding in the microstructure of dual-phase steels for sheets used in the automotive industry. ${ }^{[9]}$

The influence of microstructural bands on the global material behavior of banded and un-banded microstructures has been widely investigated in the literature with differing conclusions. The effect of such bands on local deformation and damage has been reported to be both 


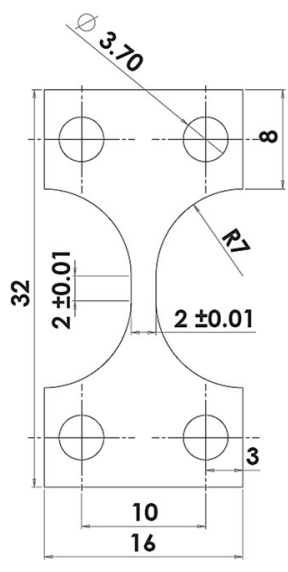

Fig. 1-Sample geometry and dimensions.

detrimental and beneficial..$^{[4,5,10-13]}$ A comparative study of two TRIP steels concluded that microstructure banding was in fact beneficial in that it resulted in ductile fracture, although it did not improve the tensile elongation. ${ }^{[6]}$ A direct comparison, however, is not straightforward as several parameters differ between the respective materials investigated, such as grain size and distribution, phase content, and inclusion morphology. ${ }^{[10,11]}$ There is therefore a need for better understanding of the effect of banding on local deformation and damage mechanisms that affect the overall mechanical performance of AHSSs.

Digital image correlation (DIC) is a technique that has been widely used as a tool to quantify the deformation at the microstructural level in advanced highstrength steels and its effect on damage. ${ }^{[14-17]}$ Several studies have been reported where in situ mechanical testing was conducted in a scanning electron microscope (SEM) for DP600 and DP1000 steel series. ${ }^{[14-17]}$ Interrupted tensile testing at small displacement intervals enabled the capture of SEM micrographs to measure strain distributions at the scale of the individual grains and phases by correlating the various images. In a recent study, the DIC technique was employed to study the effect of microstructural banding in DP600 steels. ${ }^{[18]}$ The study showed that certain limitations of the conventionally used experimental techniques, such as postmortem metallographic studies, can be overcome by an in situ approach where the mechanisms that lead to damage in such bands can be captured during tensile testing. This study revealed the detrimental effect of such bands on the micromechanical behavior of DP600 steel.

In contrast to DP steels, there is limited information and understanding of the micromechanical behavior of TRIP steels. This is partly due to there being no direct observations on the effect of microstructural bands on the micromechanical behavior of these materials. In this study, in situ micromechanical testing combined with DIC and fracture surface analysis is carried out to provide new insights into the influence of microstructural bands on the local deformation and damage mechanisms in the microstructure of TRIP steels. Unlike other studies which are usually focused on evaluating the influence of the major strain component, the minor strain distribution and evolution are also analyzed in this work in order to identify the effect of lateral contraction on the formation of microstructural bands. One of the key findings is that banding of the second-phases in the microstructure, even if it is not initially present, may be inevitable due to the inhomogeneous deformation of these steels.

\section{EXPERIMENTAL PROCEDURE}

A TRIP800 steel with an ultimate tensile strength (UTS) of $800 \mathrm{MPa}$ was studied in this work. The specimens were mechanically polished to $1 \mu \mathrm{m}$ and etched in 0.5 pct Nital solution to reveal the microstructure for SEM analysis. For EBSD analysis, to identify the distribution of retained austenite in the matrix, the specimens were mechanically polished down to $0.01 \mu \mathrm{m}$ and then electropolished with A2 electrolyte $(42 \mathrm{~V}$, 20 seconds and a temperature of $\left.8{ }^{\circ} \mathrm{C}\right) .{ }^{[19]}$ The face-centered cubic (FCC) retained austenite was differentiated from the body-centered cubic (BCC) crystal structure of ferrite, bainite, and martensite (note that the tetragonal $\mathrm{BC}$ structure of the latter was indistinguishable from the BCC).

Tensile specimens were machined, from as-received 1-mm-thick sheets, using electro-discharge wire erosion machining to minimize the effects of conventional machining on the original microstructure. The sample geometry, shown in Figure 1, has a nominal gauge area of $2 \mathrm{~mm} \times 2 \mathrm{~mm}$.

Micro-tensile testing was performed using a $5 \mathrm{kN}$ Deben testing stage inside a CamScan SEM with a crosshead speed of $1.6 \mu \mathrm{m} / \mathrm{sec}$. The tensile tests were interrupted after every $0.04 \mathrm{~mm}$ displacement increment and after the elastic stress relaxation, micrographs of the deformed microstructure were recorded. Local displacement and strain values were computed by means of DIC as explained in Reference 14. Relatively low magnification analysis (1700 times) was used in order to evaluate the effect of large microstructural defects such as microstructural bands. The micrographs were analyzed using the commercial image analysis software, DaVis 7.2 by LaVision, ${ }^{[20]}$ to determine the in-plane displacement field from which the plastic strain values were calculated. The microstructural features of the material have been directly used as patterns to perform image correlation between two successive loading steps. Micrographs of $62 \mu \mathrm{m} \times 47 \mu \mathrm{m}$ areas were used for the DIC analysis. Based on a sensitivity analysis of the grid size, a subset size of $16 \times 16$ pixels was selected for all the experimental results presented in this study. A multi-pass algorithm ${ }^{[20]}$ leading to a $1.8 \mu \mathrm{m} \times 1.8 \mu \mathrm{m}$ final interrogation window with 25 pct overlap between the windows has been used to ensure successful correlation. 
Table I. Chemical Composition (Weight Percent) of the TRIP800 Steel Studied

\begin{tabular}{cccccccccc}
\hline $\mathrm{C}$ & $\mathrm{Mn}$ & $\mathrm{Si}$ & $\mathrm{Al}$ & $\mathrm{P}$ & $\mathrm{S}$ & $\mathrm{Ti}$ & $\mathrm{Cu}$ & $\mathrm{Cr}$ & $\mathrm{Mo}$ \\
\hline 0.199 & 1.700 & 1.560 & 0.038 & 0.010 & 0.004 & 0.003 & 0.015 & 0.028 & 0.002 \\
\hline
\end{tabular}

A displacement accuracy of 0.01 pixels was obtained with a strain resolution of about 0.1 pet. $^{[20]}$

\section{RESULTS}

\section{A. Chemical Composition and Microstructural Analysis}

The chemical composition of the Si-alloyed TRIP800 is shown in Table I and the as-received microstructure and BCC-FCC phase distribution are shown in Figures 2(a) and (b), respectively. The retained austenite and martensite are further referred to as hard phases and are not differentiated in this study as the formation of the microstructural bands was independent of their individual micromechanical behavior. Similarly, the ferrite is not distinguished from any bainite that was present, but this may need to be investigated in a future study. Both micrographs show that there is a homogeneous distribution of hard phases in the as-received material, when viewed from the normal direction (ND). The banded microstructure of this material that was inherited from processing is not visible in this cross section, but is more apparent when viewed from either the transverse (TD) or rolling direction (RD). Note also in Figure 2(a), the mixed morphology of the hard phases ranges from blocks to laths.

\section{B. Stress-Strain Curve}

Figure 3 shows the stress-strain curve derived from the load-displacement measurements recorded during in situ tensile testing. It shows the macroscopic mechanical behavior of the specimen, with the drops in the curve being due to the elastic stress relaxation when the interruptions were made to take micrographs. The stress-strain response is typical for a TRIP800 with continuous yielding beginning at approximately $500 \mathrm{MPa}$ and work hardening through to the macroscopic stress magnitude of about $800 \mathrm{MPa}$. Thereafter, necking starts, but due to the relatively slow strain rate and relatively small gauge length, there is a relatively extensive post-uniform elongation region present. While the strength values correspond to a TRIP800, the strains at which necking began and the total elongation do not. The behavior up to maximum load should not be interpreted as uniform strain as in a conventional tensile test. This is because of the non-standard sample geometry that was designed to localize the deformation, such that necking and fracture would occur at a specific position to enable a series of observations at high magnifications. The observations in the text refer to the major strains $\left(\varepsilon_{\mathrm{yy}}\right)$ calculated from the crosshead extension (corrected for instrument compliance) and a gauge length of $2 \mathrm{~mm}$.

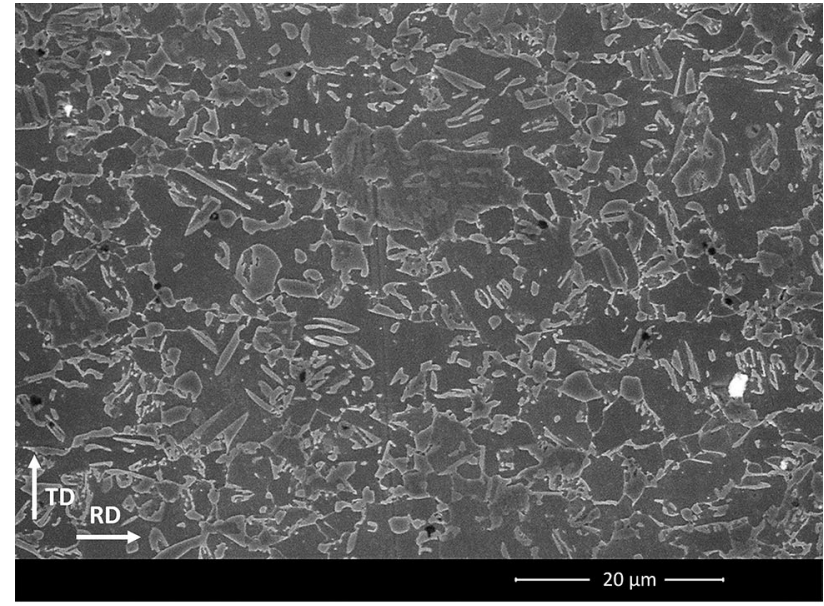

(a)

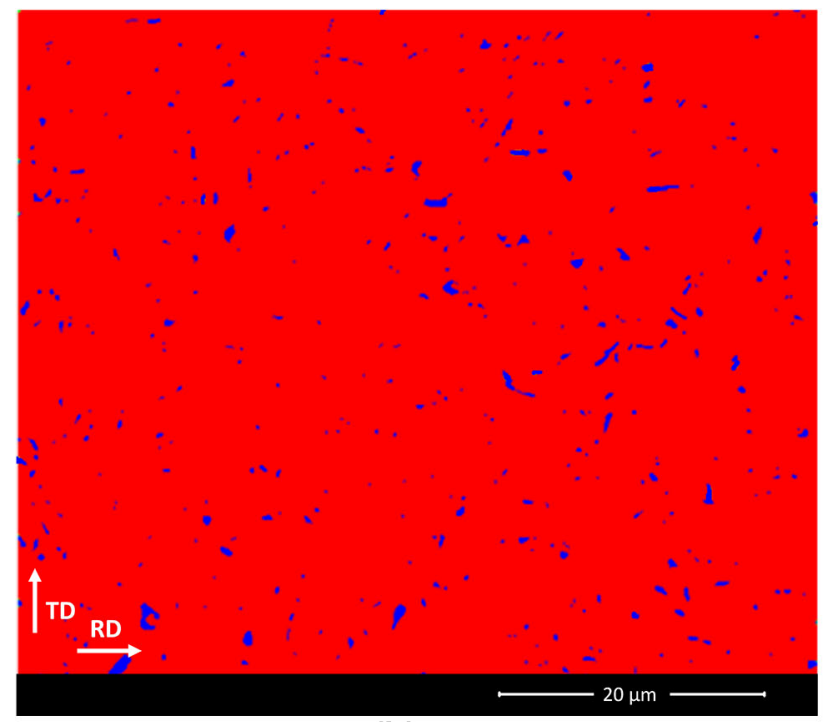

(b)

Fig. 2-(a) Micrograph showing the homogeneous distribution of the hard phases in TRIP800 steel in the RD-TD plane (i.e., rolling plane). (b) The distribution of retained austenite; the blue color corresponds to the FCC phase (retained austenite), the red color to the BCC phase (Color figure online).

\section{Digital Image Correlation Results}

In most published studies on micromechanical testing, the major strains are quantified and analyzed. ${ }^{[14-17]}$ In this study, the minor strain $\left(\varepsilon_{\mathrm{xx}}\right)$ component is first evaluated in order to assess the lateral contraction of the soft ferrite phase and its effect on the formation of microstructural bands during tensile loading. The major strains $\left(\varepsilon_{\mathrm{yy}}\right)$ are then analyzed to investigate the distribution of major strains along the hard second-phase bands. The mechanical behavior of the individual hard microstructural constituents is not analyzed in this 


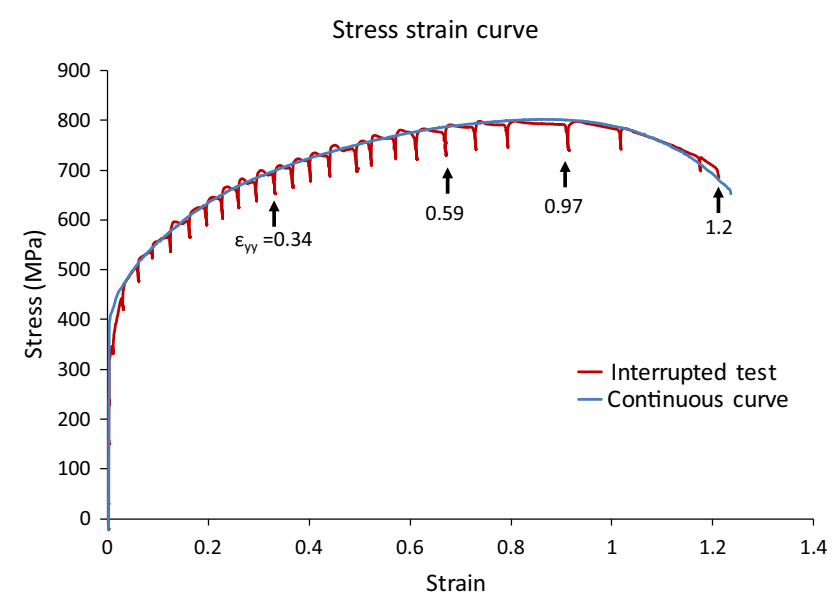

Fig. 3-Stress-strain curves for TRIP800 steel with and without interruptions. The specific interruptions where micrographs and DIC strain maps are presented in this study are highlighted.

work, as the focus of this study is to understand the mechanisms that influence the formation of microstructural bands.

\section{Minor strains $\left(\varepsilon_{x x}\right)$}

Figure $4 \mathrm{a}$ shows the selected region for the undeformed sample prior to tensile testing. The loading direction, $x$ - and $y$-axis are also indicated. Three large ferritic regions are highlighted with red broken lines in Figure 4(a). Figure 4(b) shows the same region after an applied strain of 0.34 , which corresponds to the early stage of plastic deformation, Figure 3. Figure 4(c) shows the corresponding DIC-measured minor strain $\left(\varepsilon_{\mathrm{xx}}\right)$ distribution over the area of Figure $4(\mathrm{~b})$. The strain map shows that the ferritic regions have experienced significant compressive strains along the horizontal $(x-)$ axis. The deformation of the ferrite grains along the loading $y$-axis is accompanied by extensive lateral contraction corresponding to an $\varepsilon_{\mathrm{xx}}$ strain magnitude on the order of 0.11 . It has also been shown in other studies $^{[14-17]}$ that plastic deformation initiates at ferrite grains in multi-phase advanced high-strength steels.

The ferritic regions are adjacent to small islands of hard microstructural constituents. The neighboring hard second-phase islands locally diminish the minor compressive strains. The minor strains are between $|0.1|$ and $|0.12|$ (negative in sign) within the ferritic regions, while in the neighboring hard microstructural constituents, they are between 0 and $|0.02|$ (positive in sign). The hard phases, therefore, exhibit minor strain magnitudes 6 times lower than the ferrite matrix. The highest major strains are expected to occur where the lowest lateral contraction is observed in Figure 4c.

At a higher applied strain $\left(\varepsilon_{\mathrm{yy}}=0.59\right)$, the compressive strains in the horizontal direction, resulting from the lateral contraction normal to the tensile axis, increase in the ferrite matrix from $|0.15|$ in Figure 4(c) to $|0.24|$ in Figure 4(e). The three ferrite regions of Figure 4(b) continued to elongate parallel to the loading direction in Figures 4(d), (f) and (h).
Figures 4(d) through (g) show that the strain patterns in region 1 and 2 are being disrupted by the presence of small hard phases at the yellow-arrowed regions. The presence of hard second-phases terminates and/or diverts the minor strain fields. In these two cases, the hard phases lie at a location and orientation that restricts plastic deformation along the loading axis and therefore, locally reduce the minor compressive strains in Figures 4(d) through (g).

Upon the initial stages of necking $\left(\varepsilon_{\mathrm{yy}}=0.97\right.$; Figures 4(f) and (g)) and more evidently, just before final failure, in Figures 4(h) and (i), three laterally contracted regions highlighted in red appear as bands aligned next to networks of hard second-phases and almost parallel to the loading direction. The bright features in Figure 4(h) correspond (in most cases) to the interfaces of the ferrite matrix with the hard second-phases and indicate large out-of-plane deformations. It can also be observed that the ferrite matrix has re-aligned the hard phases, creating aligned bands of second-phase islands adjacent to the ferritic regions and parallel to the loading direction. High strain gradients for the minor strain component occur in the neighborhood of these bands along the horizontal direction (minor strain axis) in Figure 4(i). Figure 4(i) shows that the zones of soft ferritic matrix experience high lateral contraction $(-)$ in between regions with near-zero compressive strains. The variation in the local strain magnitudes in the horizontal direction indicates localized and banded material flow with the highly compressed soft ferritic regions aligned next to near-zero compression bands of hard second-phases.

\section{Major strains (cyy)}

Figure 5 shows the major strain map corresponding to the micrograph of Figure 4(h) at an applied strain of $\varepsilon_{\mathrm{yy}}=1.2$ just before final fracture. Based on a simple measurement of the elongation of ferrite region in region 2 between Figures 4(a) and (h), the tensile strain value is about 150 pct and therefore, local strain values up to 220 pet seen in Figure 5 are not surprising. Similarly, for the transverse compressive strain, the wider part of region 2 gives -38 pct while the strain map using DIC shows about -40 pet at that particular location (light blue color on the scale of Figure 4(i)).

The same regions highlighted in red in Figure 4(h) are outlined in Figure 5. A comparison between Figures 4(i) and 5 shows that the evolution of mechanisms involved in the formation of microstructural bands during tensile loading is better revealed by evaluating the minor strain component. The major strain distribution map does not show any indications of banded material flow.

The highest major strains were measured within or adjacent to the microstructural bands highlighted in Figure 5. Such high strains cannot be accommodated by the hard second-phases. The two magnified insets show micro-void nucleation at the neighborhood of the hard phases, accompanied by slip band formation in the neighboring ferrite matrix (indicated by yellow arrow). Local softening occurs upon the formation of microvoids at the red-arrowed locations within the two enlarged areas in the insets. It should be noted that 

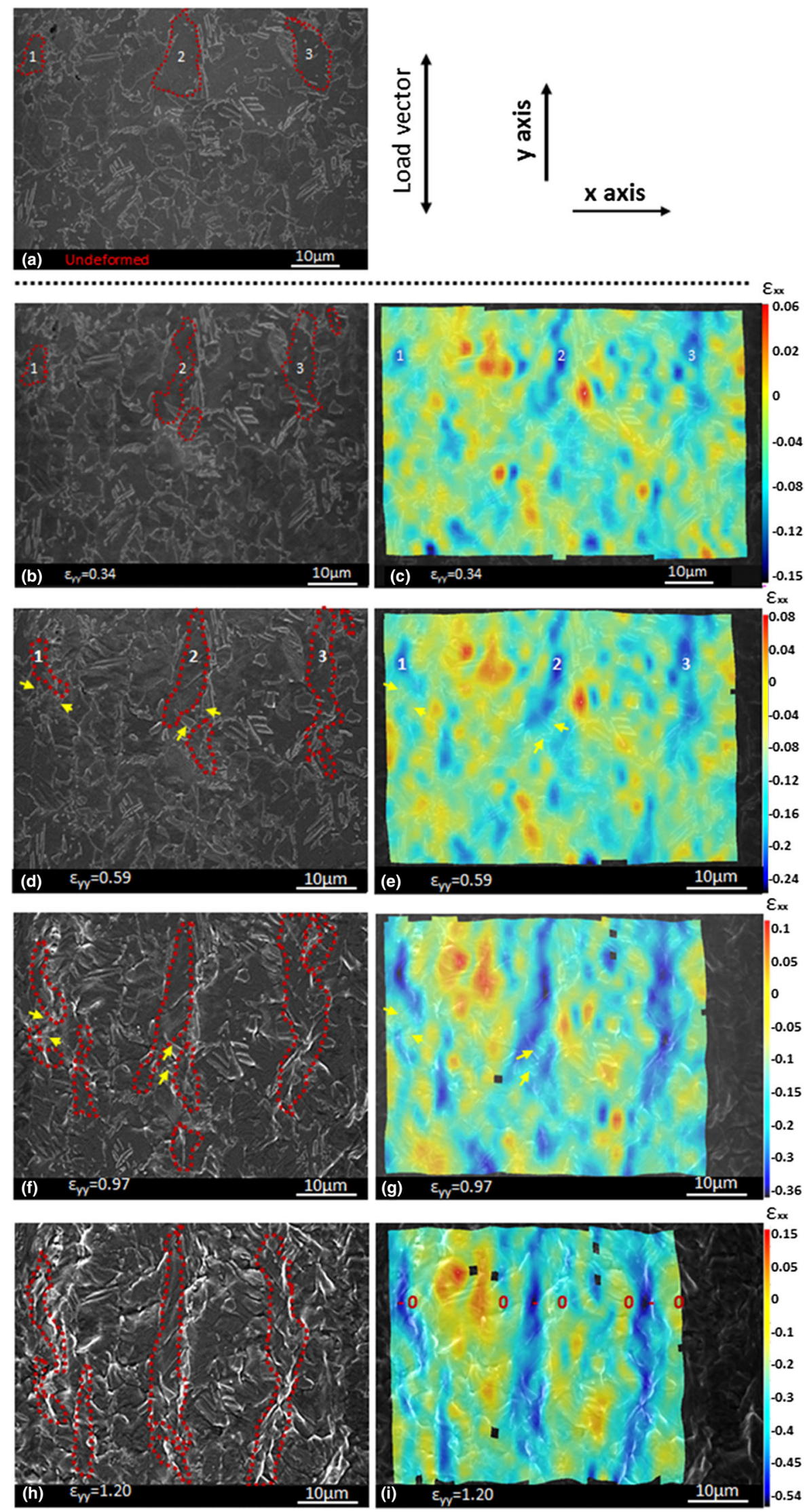

Fig. 4-Micrograph of the selected region: $(a)$ before testing (1700 times magnification); $(b),(d)$, $(f)$, and $(h)$ after specific applied strains $\left(\varepsilon_{\mathrm{yy}}\right)$ given in the sub-figures; and $(c),(e),(g),(i)$ are the corresponding $\varepsilon_{\mathrm{xx}}$ minor strain maps. This shows the developing elongation in the loading direction (vertical $/ y$ axis) and the compression along the horizontal $/ x$ axis of the ferrite matrix. 


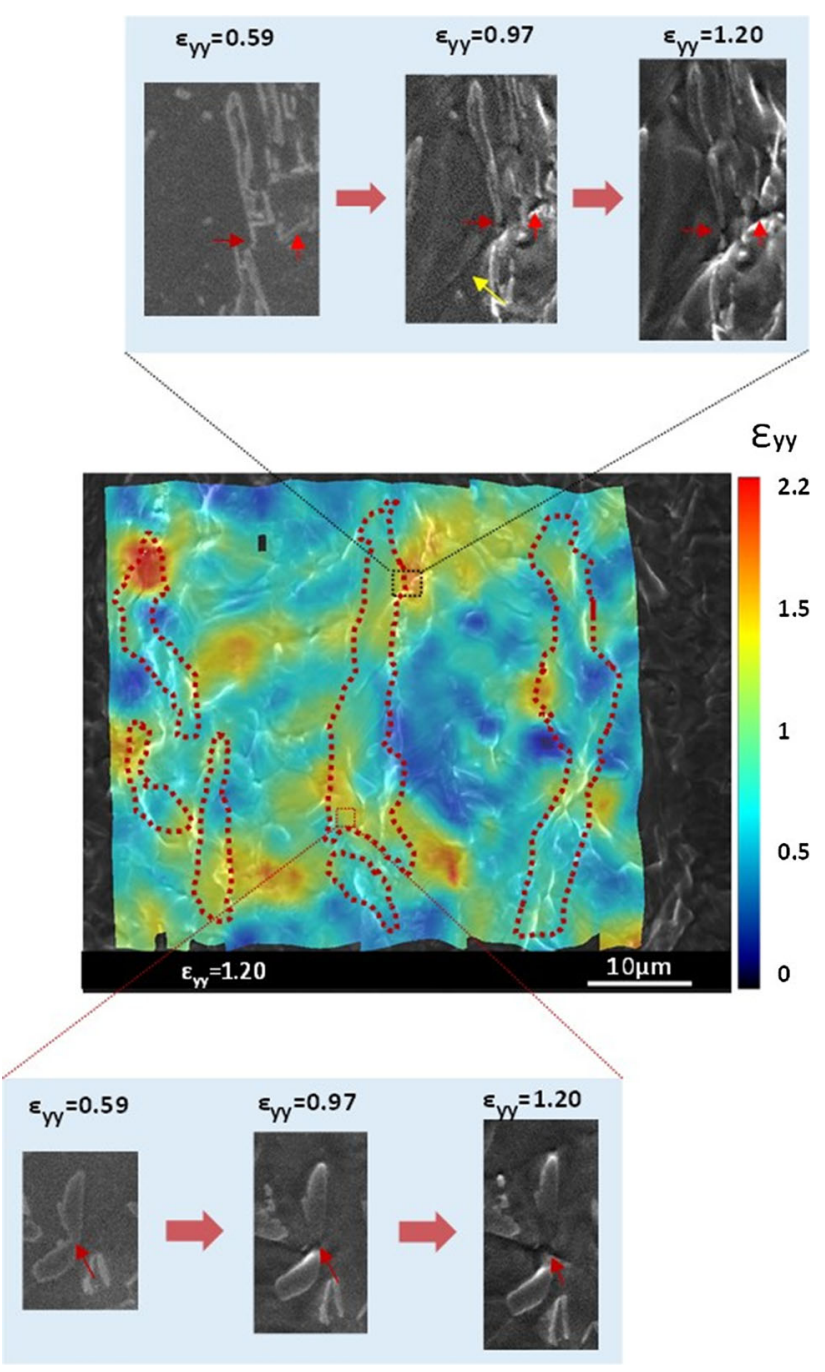

Fig. 5-The corresponding major strain map $\left(\varepsilon_{\mathrm{yy}}\right)$ for an applied strain of $\varepsilon_{\mathrm{yy}}=1.2$. The insets show enlargements of regions where micro-void nucleation has occurred at the arrowed locations, which correspond to highly strained regions with hard phase microstructural constituents.

strain values calculated through DIC in regions of the microstructure where damage appears during deformation are inaccurate and can only be considered qualitatively due to a local loss of correlation, associated with a sudden change of contrast in the interrogation window at the damage location. The algorithm used by the DaVis software to return a strain value at these locations is unknown. There are, however, regions of very high strains where voids nucleated, separated by low-strained, undamaged regions along the red-outlined areas. Figure 5 reveals that the gradients for the major strain component are larger along the contiguous bands of hard second-phases. These large (major) strain gradients suggest that the alignment of the soft ferrite matrix adjacent to the contiguous bands of hard second-phases leads to localized damage at specific sites in between the hard phases along these bands.

\section{Mid-plane Analysis}

The fractured specimens, shown schematically in Figure 6(a), were sectioned along the mid-plane (black-dotted line) relative to the specimen width. The ND-RD plane of the cut samples was metallographically polished and etched with 0.5 pct Nital solution. An undeformed and a heavily deformed region of the sample (region A and B, respectively, in Figure 6(b)) were selected and analyzed using SEM imaging to evaluate the effect of tensile loading on the formation of microstructural bands in a conventional post-mortem approach.

In the as-received microstructure (Region A) shown in Figure 6(c), the hard second-phases decorate the ferrite grain boundaries and in many cases form contiguous networks, as highlighted by the red arrows. These features become even more apparent with deformation as shown in Figures 6(d) and (e). In this type of microstructural band, it is not simply the hard second-phases that are aligned, but the ferrite grain boundaries that they lie upon are as well.

The undeformed microstructure also exhibits equiaxed ferrite grains (delineated by the grain boundary networks), but near the fracture surface they become significantly elongated along the loading direction (i.e., RD) and compressed in the normal direction. In the heavily deformed region near the fracture surface (region B) shown in Figures 6(d), (e) and (f), many of the grain boundary networks have become aligned with the load axis, along with the individual lath-shaped (possibly plates in cross section) hard second-phases, to form bands with an interspacing of 2 to $5 \mu \mathrm{m}$.

Large voids ranging 5 to $15 \mu \mathrm{m}$ in size are clearly visible in Figure 6(d). They are all associated with oxide inclusions that will be described further in the next section on the fractography. Otherwise, it is remarkable how damage tolerant the majority of the contiguous grain boundary networks (CGBNs) are on the basis of the lack of visible voids, especially with the obvious high levels of ferrite deformation that have occurred. Some voids are present in the constrained ferrite between the hard phases, but the majority are along certain grain boundary networks. These voids are indicated by arrows in Figures 6(e) and (f), which show the CGBN in the deformed microstructure at higher magnification.

Figure 6(c) shows that in the as-received microstructure (Region A) there are pre-existing horizontal CGBNs that extend over the whole width of the micrograph, indicated by red arrows. The initial spacing between these microstructural bands is approximately 40 to $70 \mu \mathrm{m}$, but in the heavily deformed part of the specimen these features become approximately $10 \mu \mathrm{m}$ apart, as shown in Figure 6(d). These CGBNs lie parallel to the loading direction and develop a large number of voids along them, even up to 100 micrometers away from to the fracture surface. Figure $6 f$ shows that these CGBNs correspond to the fissures extending from the fracture surface, where the crack appears to propagate along the hard second-phases and the adjacent ferrite matrix. 


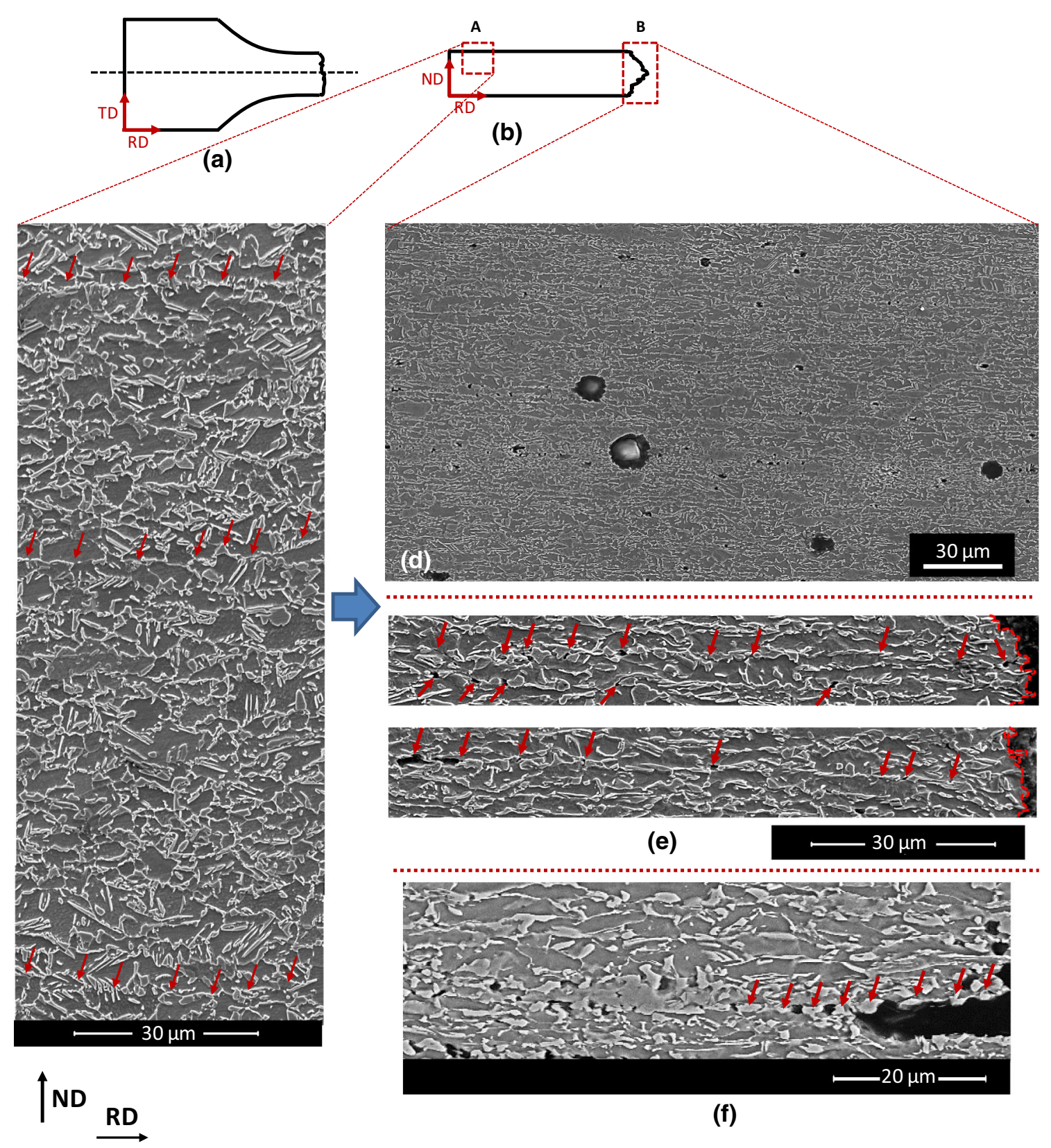

(c)

Fig. 6-(a) Schematic of the fractured tensile sample (looking in the ND direction) showing the mid-width ND-RD plane sectioning (black-dashed line) for post-mortem analyses, $(b)$ schematic looking in the TD direction showing two red-boxed regions corresponding to the undeformed and deformed regions for SEM analysis, $(c)$ micrograph of the relatively undeformed material and $(d)$ through $(f)$ micrographs of the heavily deformed material close to the fracture surface. Red arrows in Figures (c), (e), and (f) highlight the CGBNs (Color figure online).

\section{E. Fracture Surface Analysis}

SEM micrographs of the fracture surface of the sample are shown in Figure 7. The most obvious features seen in the lower magnification image (Figure 7(a)) are the fissures aligned with the transverse direction. Some of these appear quite deep (indicated by white arrows) and several of them extend over the whole sample width $(2 \mathrm{~mm})$. The spacing in the thickness direction between them varies from 40 to $100 \mu \mathrm{m}$. In between the larger fissures, there is also a parallel arrangement in surface topography giving the impression of layers aligned to the rolling plane (Figures 7(d) through (f)). These have a spacing of about $5 \mu \mathrm{m}$, which notably corresponds approximately to that of the microstructural bands of hard phases in region of fracture shown in Figure 6. Some of these linear features are smaller fissures that are visible at higher magnifications.

At the higher magnification (Figures 7(b) through (e)), it is clear that the fracture surface consists of 


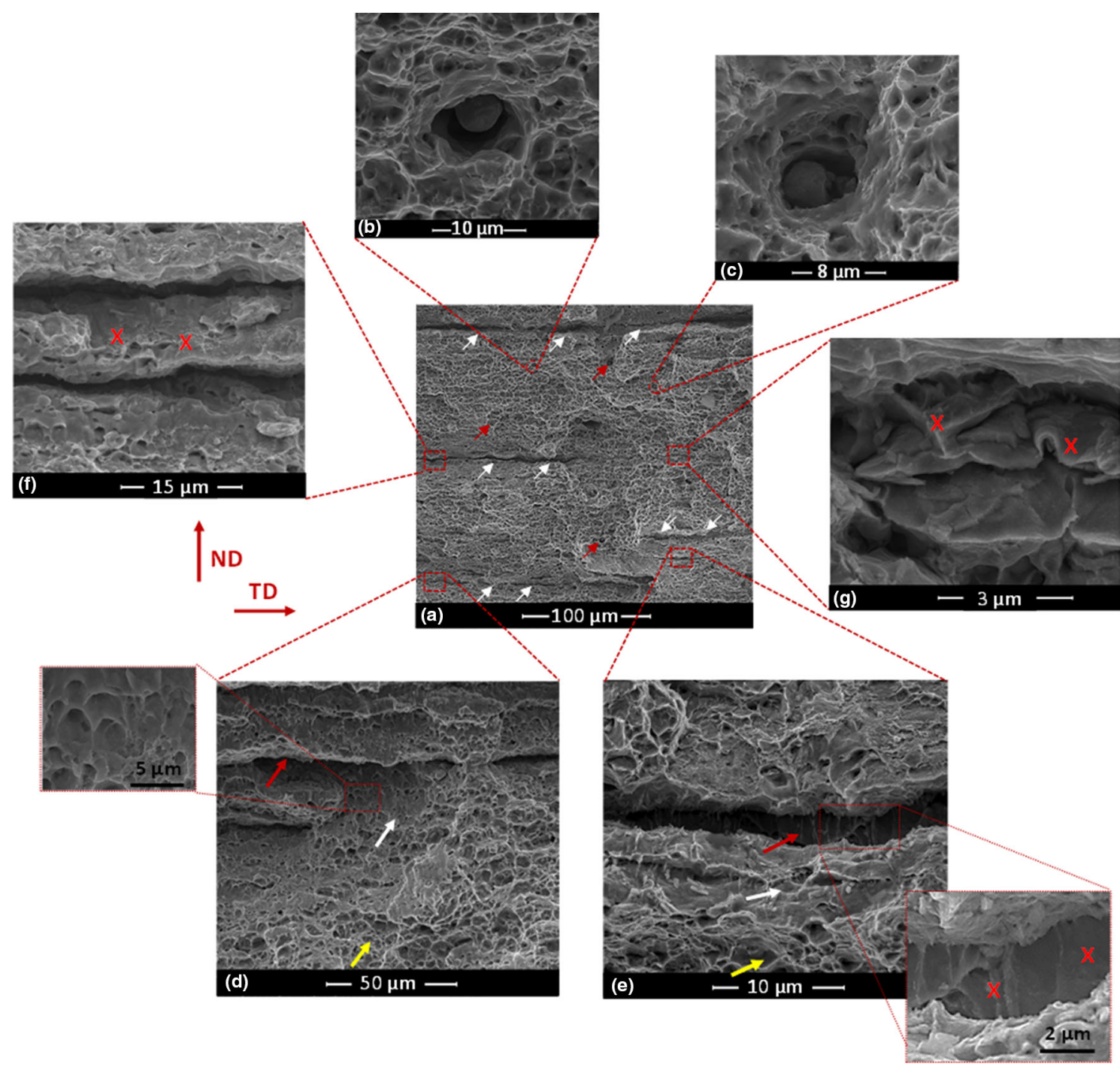

Fig. 7-SEM micrographs of the fracture surface: $(a)$ low-magnification image showing an overview and high-magnification images showing (b) through $(c)$ voids at inclusion sites, $(d)$ through $(g)$ dimpled and shear surface topography near and within fissures.

dimples indicative of ductile rupture. The majority of the dimples in the matrix are less than $5 \mu \mathrm{m}$ in diameter and relatively symmetrical. Even within the large fissures, there are small $(<5 \mu \mathrm{m})$ dimples (Figure $7(\mathrm{~d})$ and enlarged region) that are asymmetric, which is suggestive of shear. There are some larger dimples ranging in size from 5 to $10 \mu \mathrm{m}$, many of which contain inclusions. Most of these inclusions are spherical (Figures 7(b) and (c)) and EDX analyses showed to contain significant amounts of $\mathrm{O}, \mathrm{Al}$, and $\mathrm{Ca}$ (see Figures 8(a) through (b)) as well as $\mathrm{Cu}, \mathrm{Mn}$, and $\mathrm{S}$, indicative of oxides and sulfides that are ubiquitous in steels.

It is not possible to identify or distinguish on the fracture surface the ferrite matrix and the hard phase islands. It can be reasonably assumed that all of the austenite in this TRIP near the rupture has transformed into high-carbon martensite. One might expect that this microstructure constituent would be recognizable on the surface as regions of classical transgranular brittle fracture with the characteristic flat facets exhibiting river-lines. However, no such regions could be found in this fracture surface.

It is shown in Figures 7(d) and (e) that there are regions of the surface that are relatively smooth and free of dimples within (red arrows) and adjacent (white arrows) to the large fissures. Some studies have interpreted such regions as brittle or cleavage failure. ${ }^{[21-23]}$ However, the non-planar topography of these regions is more indicative of shear-band failure or ductile tearing at a crack tip. Such regions are indicated by $\mathrm{X}$ in the enlarged region of Figure 7(e).

The only obviously brittle topographic features were observed in some smaller fissures like that shown in Figure $7(\mathrm{~g})$. Some of these are also visible lying on the dimple-free regions in Figure 7(e), but they are present in insufficiently high number density to be responsible for the whole fissure. These are thin plates several tens to hundreds of nanometers thick. EDX analyses showed S, 

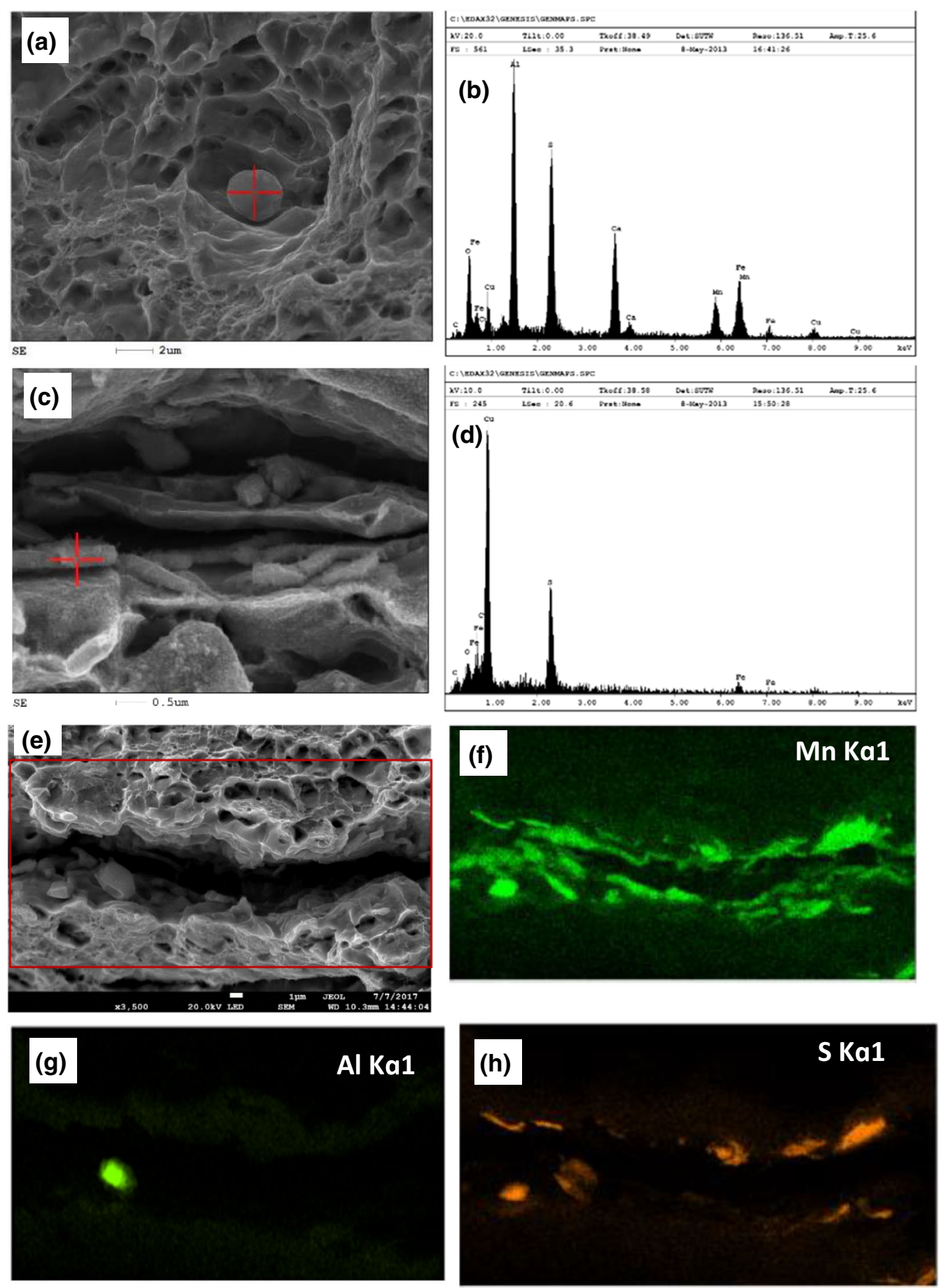

Fig. 8- SE micrographs and the corresponding EDX analyses of inclusions on the fracture surface. The spectra for point analyses on inclusions (shown in $(a)$ and $(c))$ are given in $(b)$ and $(d)$. The composition maps in $(f)$ to $(h)$ are for the fissure shown in sub-figure $(e)$.

$\mathrm{Mn}$, and/or $\mathrm{Cu} \mathrm{K}$-alpha peaks (see Figures 8(c) through (d) for a $\mathrm{Cu}-\mathrm{S}$ example), showing them to be sulfide inclusions $(\mathrm{MnS} / \mathrm{CuS})$. Such features are also ubiquitous in steels, especially those with relatively high Mn-levels and are thin $(\mathrm{Mn}, \mathrm{Cu}) \mathrm{S}$ stringers. EDX maps were made of some regions at the fissures such as that shown in Figures $8(\mathrm{e})$ through $(\mathrm{g})$. These showed that while the presence of S always correlated to presence of $\mathrm{Mn}$, there were some regions where only Mn was measured. This may be a consequence of the rough topography of the fracture surface interfering with the detection of a light element such as $\mathrm{S}$.

In summary, there are three main features: ductile dimples within the matrix, micro-voids containing inclusions (Figures 7(b), (c), and (g)), and fissures at the white-arrowed locations, extending into the sample from the fracture surface. 


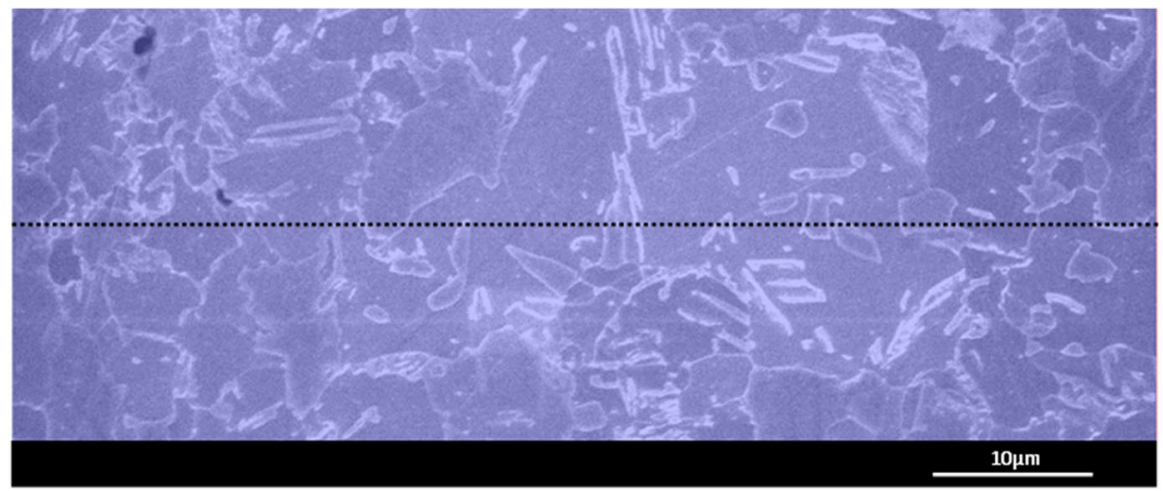

(a)

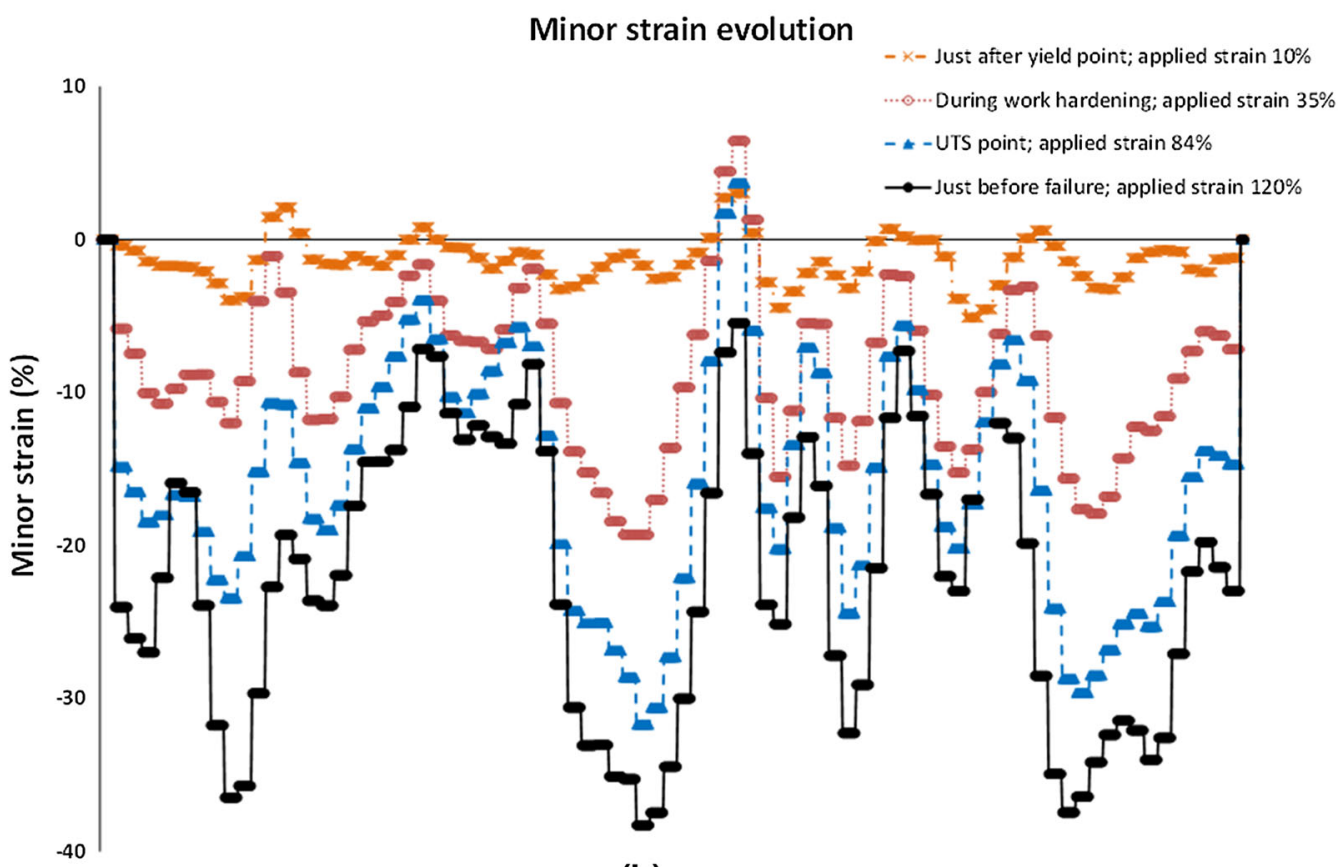

(b)

Fig. 9- The evolution of the minor strain during the tensile test taken along the dotted line shown in micrograph $(a)$, and $(b)$ the minor strains at (i) just after yielding, (ii) during work hardening, (iii) at UTS point and (iv) just before failure.

\section{DISCUSSION}

While microstructure banding is a common feature of steels, the TRIP800 material studied here had initially a relatively homogeneous distribution of second-phase, albeit along the ferrite grain boundaries. The only feature resembling the classical type of banding was a more subtle structure described here as horizontal continuous grain boundary networks (CGBNs), highlighted in Figure 6(c). CGBNs are probably like conventional microstructure bands; in that, they are a consequence of chemical segregation in combination with the elongated austenitic grain morphology produced in hot-rolling and annealing stages. In both the in situ and post-mortem observations taken from different directions, the distribution of hard second-phases became so inhomogeneous with deformation that it could be described as banding. The importance of the horizontal CGBNs that were originally present is made apparent by their correlation to the fracture surface topography, which is a post-mortem aspect of this study. However, it is the in situ experiments that give insights to how and why these features have developed during the deformation and these results will be discussed first.

\section{A. Strain Evolution at the Microstructural Level}

The Digital Image Correlation results shown in Figure 4 reveal that the material forms macroscopic bands of hard second-phases during tensile loading. This is in agreement with the observations made in Figure 6, where the spacing between microstructural bands decreases due to the contraction of the sample in the thickness direction (i.e., ND).

Evaluation of the distribution and evolution for the minor strain component reveals that the mechanism of formation of these hard phase zones is primarily the lateral contraction of the soft ferrite phase (Figure 4). 
High compressive strains for the minor strain component are initially distributed within the ferritic regions (Figures 4(b) and (c)). This is also found in other studies where the highest major strains are measured within the soft ferrite matrix. ${ }^{[1-17]}$ These regions of high compressive straining spread to neighboring regions along the loading direction by rotating and aligning the hard phases adjacent to the large ferrite grains as shown in Figures 4(b) through (i). More specifically, the strain patterns in regions 1 and 2 of Figure 4(c) are interrupted at the arrowed locations due to the presence of relatively large hard phase islands in line with the large ferrite regions. In order to accommodate plastic deformation and circumvent the hard phases impeding the further deformation of the ferrite matrix, the compressive strains divert towards different directions. This is favored by the formation of slip bands in the soft ferrite matrix at angles close to $45 \mathrm{deg}$ with respect to the loading axis. Just before final failure, the soft ferrite matrix has displaced the hard phases and the minor strain fields have become aligned almost parallel to the loading direction in Figure 4(i). It is suggested here that diverting the local strain fields is beneficial for the alloy, as more energy would be required to align the hard islands parallel to the loading direction, delaying further the onset of damage nucleation. Distributing the hard second-phases evenly within the matrix, not just along grain boundaries, but also within the interior of the ferrite grains, can increase the deformability of the alloy in tension.

At the last stage of the test, the regions of high compressive strains in Figure 4(i) extend throughout the whole microstructural region in the loading direction. A 'banded material flow' can thus be transferred to much larger distances with respect to the local scale of the micrograph (50 $\mu \mathrm{m}$ length) of Figure 4(i). This is in agreement with the findings from the mid-plane analysis of Figure 6(d), where it is shown that the hard phase bands have voids as far as $100 \mu \mathrm{m}$ from the fracture surface. It was observed (Figure 4(i)) that along the horizontal axis, the strains were near-zero (0) along the hard second-phase bands, highly negative $(-)$ along the soft ferrite matrix and again near-zero (0). The observations suggest the occurrence of localized plastic flow along the soft ferrite bands in contrast to the restricted deformation of the hard phase bands. Comparing the two strain maps of Figures 4(c) and (i), at the first stages of plastic deformation the strain patterns appear more evenly distributed over the microstructure, while just before failure there is a well-defined strain distribution of compression-tension-compression alterations parallel to the loading axis.

This is better illustrated in Figure 9, where the evolution of the minor strains along a line on the DIC strain map in Figure 9(a) was obtained at four different stages of the test. The evolution of the minor strains is shown at four stages during the test in Figure 9(b); just after yielding $(0.1)$, during work hardening $(0.35)$, at UTS point (0.84) and just before failure (1.25). Even at the first stages of plastic deformation, the material has started forming microstructural bands. Most of the microstructural rearrangements occur during plastic deformation up to the UTS point. Consequently, it is during work hardening that the material forms these bands by redistributing the phases in the matrix. This can be seen by the considerable increase of the compressive strains between the yield point and UTS point. It is suggested here that an optimization of the distribution of the hard phases in the matrix could potentially increase the deformation capability of the alloy. The tendency to form microstructural bands of hard second-phases could, in turn, be reduced, even from the first stages of plastic deformation.

Further plastic deformation of the soft ferritic regions leads to damage nucleation in between the hard phases and at the adjacent second-phase bands. The voids that formed in between these hard second-phases have an average spacing of 5 to 15 microns between them along each band (Figure 6(d)). There is a sequence of damaged and undamaged regions along each band. This is in agreement with the major strain distributions measured in Figure 5. Alternating regions of high and low major strains can be seen along and adjacent to the hard phase bands (Figure 5). This is due to the fact that regions of high strain localizations and micro-void nucleation are interrupted by relatively low-strained undamaged regions. It is noted here the much higher maximum tensile strain values recorded on the strain map (220 pct) in Figure 5 in comparison to maximum values up to 130 pct in DP steels recorded in other studies. ${ }^{[16,24]}$ The higher local major strains of this TRIP steel can be related to the formation of the microstructural bands and the extensive shape changes that take place within the soft ferritic regions (Figures 4(b), (d), (f), and (h)).

Comparing Figures 4 and 5 for the minor and major strain maps, it can be concluded that the latter does not reveal the banded material flow. The major strain distribution is similar to the strain maps shown in other studies on DP steels, ${ }^{[14-17]}$ where the high major strains are oriented at an angle (close to $45 \mathrm{deg}$ ) with respect to the loading direction due to localized slip and shear bands.

In summary, the sequence of deformation and damage nucleation mechanisms, with reference to the macroscopic stress-strain curve, is as follows:

1. During the first stages of macroscopic plastic deformation, the ferrite matrix deforms and in particular elongates in a way that aligns the grain boundary networks of second-phases to the loading direction.

2. At the latter stages of work-hardening, the ferrite regions rotate along with the hard phases adjacent to them, in order to achieve further plastic deformation, which results in alternating bands of ferrite and hard second-phase. The DIC observations in Figure 4 suggest that the alignment of the hard phases adjacent to the large ferrite grains facilitates plastic deformation in the ferritic matrix as there are no hard phases restricting plastic slip.

3. In the post-uniform stages, further stretching-straining of the ferrite between the hard second-phases leads to damage nucleation (voids) 
within the band, primarily occurring between co-joined hard second-phase islands.

\section{B. Correlation to Mid-plane \& Fracture Surface Analyses}

The in situ experiments in conjunction with DIC measurements were all based on observations of the rolling plane (TD-RD), but the post-mortem observations of the longitudinal cross section (ND-RD) also revealed microstructural banding. Some of this was already present before deformation, as seen in the undeformed part of the sample (Figure 6(c)) as horizontal CGBNs that probably formed during the thermomechanical processing of the material. ${ }^{[18]}$ However, by comparing the undeformed microstructure (region $\mathrm{A}$ ) with that of the heavily deformed (region B) in Figure 6, the majority of this banding developed as a consequence of the deformation mechanisms described in the previous section.

The CGBNs of hard second-phases play a critical role in damage nucleation and evolution. The voids shown in Figure 6(d) suggest that damage can be transferred along them, but it is the longer horizontal networks that were already exiting in the as-received material that are critical. These are already aligned with the loading axis and are the first to nucleate micro-voids as the adjacent ferrite matrix elongates. The early failure of these regions is revealed by voids observed along them as far as $100 \mu \mathrm{m}$ from the fracture surface. This again is in accordance with the observations from DIC, where the highly strained zones were found to extend over the whole microstructural region in Figure 4(i), a length of approximately $50 \mu \mathrm{m}$.

The low-magnification micrograph of the fracture surface shows that there was an array of features aligned with TD with a spacing of about $5 \mu \mathrm{m}$. This corresponds to the band interspacing of the re-aligned CGBNs at the deformation level near the fracture surface. On the basis of the width of the larger fissures that extend hundreds of micrometers to even almost the whole of the sample (Figure 7(a)), it seems more likely that the pre-existing horizontal CGBNs are responsible as opposed to inclusions. However, while the majority of inclusions were globular in shape, some were thin plates that would be good initiation sites and propagation paths. Thinplate sulfide type inclusions were observed on some of the fissure surfaces, but since inclusions and CGBN's are related to alloying micro-segregation, the co-location of both is expected. Nevertheless, the inclusions and the (spherical) voids that formed around them at and near the fracture surface, were of the order of 5 to $15 \mu \mathrm{m}$ and thus, the length scale of the fissures and fracture surface topography (hundreds of micrometers) correlate better to pre-existing CGBNs.

Adjacent to and within the fissures, the matrix is sheared at a direction close to $45 \mathrm{deg}$ with respect to the load direction. This can be seen from the increasing ductile dimple asymmetry toward the fissures, which is indicative that the direction for the local highest strains (and strain vectors) is different from the load vector and is increasingly shear-like. This could be a result of the bands of hard phases changing the deformation mode of the intermediary ferritic matrix into localized plastic shear because of geometrical constrains.

The above post-mortem observations are taken from the ND-RD cross section (Section III-D) and the fracture surface (Section III-E), which is approximately a ND-TD plane view. The shear process was also observed in situ in the RD-ND plane view (Section III-C-1). In Figure 5, it was found that next to the hard phase islands, slip bands formed at the yellow-arrowed location within the neighboring ferrite region, close to $45 \mathrm{deg}$ with respect to the loading direction. The occurrence of damage nucleation is shown more evidently in the top part of Figure 5, which shows micro-void nucleation in a network of small hard phases close to each other. Damage nucleation at the hard phase band is accompanied by deformation localization by slip band formation in the neighboring ferrite grain at an angle close to $45 \mathrm{deg}$ with respect to the loading direction. It appears that damage nucleation within the network of hard phases is followed by shear deformation mechanisms within the neighboring ferrite matrix. This is due to the deformation mechanisms of the soft ferrite phase that occurs primarily by the formation of homogeneous plastic slip and inhomogeneous plastic strain localization due to slip band formation. The latter occurs at angles close to $45 \mathrm{deg}$ angle with respect to the loading direction which decreases the size and number of dimples (Figure 7(d)).

A key aspect of the mechanism described above is that it is predominantly the ferrite matrix that ultimately fails and propagates the final rupture and side fissures. This correlates to the absence of brittle features on the fracture surface, aside from inclusions, with the predominance of ductile dimple rupture in tensile or shear mode and localized shear failure in the fissures. There have been other fractography investigations of DP and TRIP steels that have observed regions of cleavage facets, ${ }^{[25-29]}$ but also studies ${ }^{[30-35]}$ where the entire fracture surface consists of dimples. This study's observations correspond with those of the latter, such that either the ruptured martensite also exhibits ductile dimples or the fracture surface only results from the rupture of the ferrite matrix.

\section{Summary}

The damage initiation and evolution of the TRIP steel investigated are not fundamentally different to that of DP-steels. The first cracks and voids to form are associated with the hard phase in this TRIP steel, just as it is for DP-steels. Note that it is well established that in conventional TRIP steels, the majority of austenite transforms into martensite in the early stages of plastic deformation such that well before the UTS is reached, only a few volume percent remains. In the current study, aside from the initial microstructure, the microstructural observations start a few percent before the UTS. ${ }^{[36-38]}$ No differential behavior was observed in different individual islands of hard phases and if austenite was present, it was not distinguishable in terms of 
deformation behavior. Nevertheless, while a detailed characterization of microstructure constituents is not given here, it would be a useful separate study in itself, like that performed in related material. ${ }^{[39,40]}$

Despite the hard phase forming an almost contiguous network around the ferrite grains, the vast majority of the deformation occurred in the relatively soft ferrite matrix phase. The ferrite elongates in the direction of the loading direction while contracting laterally and in the thickness direction, due to plastic incompressibility. The lateral contraction of the soft ferrite phase leads to the formation of microstructure bands of soft ferrite phase adjacent to bands of hard second-phases. This results in strain localization and void formation along the CGBNs, particular those that were already present in the starting microstructure and aligned with the load axis. Voids formed in the ferrite between the hard phases and by fracture of the individual islands within the CGBNs. Void formation along the CGBNs is due to two mechanisms: the extensive elongation that occurs in the neighboring large ferrite grains and the strain localization in between the hard phases. Deformation during these stages propagates at an angle close to $45 \mathrm{deg}$ with respect to the loading axis towards the neighboring ferrite matrix.

In the final stages leading to fracture, the voids along CGBNs coalescence to form fissures. The deformation mode becomes increasingly of shear-mode that leads to localized slip failure. It is likely that the fissures grow to the point that ductile rupture occurs between them.

The damage and final rupture mechanism described above may explain the observations in another investigation of two TRIP steel materials with and without banding. It was concluded that banding of martensite leads to the presence of massive ferrite regions that were thought to be more easily deformable with respect to geometrically constrained ferritic regions confined by hard martensite islands in the steel with non-banded martensite morphology. ${ }^{[6]}$

\section{CONCLUSIONS}

In dual-phase microstructures consisting of a soft matrix and hard second-phases, banding may be inevitable due to the inhomogeneous deformation, even if it is not initially present. Only a subtle microstructural band of hard phases was observed in this as-received TRIP800, in the form of horizontal contiguous grain boundary networks, aligned with the rolling plane. Additional bands of hard phase and soft-phases aligned parallel to the loading direction developed during tensile deformation. This was a consequence of lateral contraction of the soft ferrite phase. The pre-existing contiguous grain boundary networks of hard second-phases were of greater importance to final fracture than inclusions. These bands were where the first voids nucleated and grew along them at sufficient densities to coalesce and develop into fissures that eventually led to final ductile rupture. It is suggested that minimizing the presence of CGBNs of hard second-phases in the initial microstructure will increase the formability.

\section{ACKNOWLEDGMENTS}

This research was carried out under Project Number M41.2.10398 in the framework of the Research Program of the Materials innovation institute-M2i (www.m2i.nl). The authors would also like to thank Tata Steel R\&D, IJmuiden in the Netherlands for providing the material of this study. PE and BAS would also like to thank funding from Engineering and Physical Sciences Research Council (Grant EP/M014096/1).

\section{OPEN ACCESS}

This article is distributed under the terms of the Creative Commons Attribution 4.0 International License (http://creativecommons.org/licenses/by/4.0/), which permits unrestricted use, distribution, and reproduction in any medium, provided you give appropriate credit to the original author(s) and the source, provide a link to the Creative Commons license, and indicate if changes were made.

\section{REFERENCES}

1. G. Thomas and J.Y. Koo: Design of Duplex Low Carbon Steels for Improved Strength: Weight Applications, Lawrence Berkeley National Laboratory, Berkeley, 1977, p. 40.

2. M. Sarwar and R. Priestner: J. Mater. Sci., 1996, vol. 31, pp. 2091-95.

3. J.D. Verhoeven: J. Mater. Eng. Perform., 2000, vol. 9, pp. 286-96.

4. X.L. Cai, J. Feng, and W.S. Owen: Metall. Trans. A, 1985, vol. 16, pp. $1405-15$.

5. A.R. Marder: Metall. Trans. A, 1982, vol. 13, pp. 85-92.

6. A. Ramazani, H. Quade, M. Abbasi, and U. Prahl: Mater. Sci. Eng. A, 2016, vol. 651, pp. 160-64.

7. J.H. Whitely: J. Iron. Steel. I., 1926, vol. 113, p. 213.

8. C.F. Jatczak, D.J. Girardi, and E.S. Rowland: Trans. ASM., 1956, vol. 48, pp. 279-305.

9. F.G. Caballero, A. García-Junceda, C. Capdevila, and C.G. de Andrés: Mater. Trans., 2006, vol. 47, pp. 2269-76.

10. A. Sakir Bor: ISIJ Int., 1991, vol. 31, pp. 1445-46.

11. R.A. Grange: Metall. Trans., 1971, vol. 2, pp. 417-26.

12. M. Mazinani and W.J. Poole: Metall. Mater. Trans. A, 2007, vol. 38A, pp. 328-39.

13. W.A. Spitzig: Metall. Trans. A, 1983, vol. 14, pp. 471-84.

14. H. Ghadbeigi, C. Pinna, and S. Celotto: Exp. Mech., 2012, vol. 52, pp. $1483-92$.

15. H. Ghadbeigi, C. Pinna, and S. Celotto: Mater. Sci. Eng. A, 2013, vol. 588 , pp. $420-31$.

16. H. Ghadbeigi, C. Pinna, S. Celotto, and J.R. Yates: Mater. Sci. Eng. A, 2010, vol. 527, pp. 5026-32.

17. J. Kang, Y. Ososkov, J.D. Embury, and D.S. Wilkinson: Scr. Mater., 2007, vol. 56, pp. 999-1002.

18. C.C. Tasan, J.P.M. Hoefnagels, and M.G.D. Geers: Scr. Mater., 2010, vol. 62, pp. 835-38.

19. S. Zaefferer, P. Romano, and F. Friedel: J. Microsc., 2008, vol. 230, pp. 499-508.

20. DaVis Strain Master Software LAVision (2005). 
21. J.H. Kim, D. Kim, H.N. Han, F. Barlat, and M.G. Lee: Mater. Sci. Eng. A, 2013, vol. 559, pp. 222-31.

22. J. Samei, D.E. Green, J. Cheng, and M.S. de Carvalho Lima: Mater. Des., 2016, vol. 92, pp. 1028-37.

23. J. Zhang, H. Di, Y. Deng, and R.D. Misra: Mater. Sci. Eng. A, 2015, vol. 627, pp. 230-40.

24. S. Hazra, P. Efthymiadis, D. Proprentner, B. Shollock, and R. Dashwood: Procedia Eng., 2017, vol. 207, pp. 1314-19.

25. Q. Lai, O. Bouaziz, M. Gouné, L. Brassart, M. Verdier, G. Parry, A. Perlade, Y. Bréchet, and T. Pardoen: Mater. Sci. Eng. A, 2015, vol. 646, pp. $322-31$.

26. H.S. Lee, B. Hwang, S. Lee, C.G. Lee, and S.J. Kim: Metall. Mater. Trans. A, 2004, vol. 35A, pp. 2371-82.

27. U. Donatus, K.K. Alanem, and M.I. Momoh: J. Phys. Sci., 2016, vol. 27, pp. 103-10.

28. S. Papaefthymiou, U. Prahl, W. Bleck, S. van der Zwaag, and J. Sietsma: Int. J. Mater. Res., 2006, vol. 97, pp. 1723-31.

29. U. Prahl, S. Papaefthymiou, V. Uthaisangsuk, W. Bleck, J. Sietsma, and S. van der Zwaag: Comput. Mater. Sci., 2007, vol. 39, pp. 17-22.

30. H. Ashrafi, M. Shamanian, R. Emadi, and N. Saeidi: Mater. Sci. Eng. A, 2017, vol. 680, pp. 197-202.
31. B. Ravi Kumar, N.K. Patel, K. Mukherjee, M. Walunj, G.K. Mandal, and T. Venugopalan: Mater. Sci. Eng. A, 2017, vol. 685, pp. 187-93.

32. K.K. Chawla, P.R. Rios, and J.R.C. Guimarães: J. Mater. Sci. Lett., 1983, vol. 2, pp. 94-98.

33. A. Das, M. Ghosh, S. Tarafder, S. Sivaprasad, and D. Chakrabarti: Mater. Sci. Eng. A, 2017, vol. 680, pp. 249-58.

34. A.G. Kalashami, A. Kermanpur, A. Najafizadeh, and Y. Mazaheri: Mater. Sci. Eng. A, 2016, vol. 658, pp. 355-66.

35. A. Ramazani, A. Schwedt, A. Aretz, U. Prahl, and W. Bleck: Comput. Mater. Sci., 2013, vol. 75, pp. 35-44.

36. B.L. Ennis, E. Jimenez-Melero, E.H. Atzema, M. Krugla, M.A. Azeem, D. Rowley, D. Daisenberger, D.N. Hanlon, and P.D. Lee: Int. J. Plast., 2017, vol. 88, pp. 126-39.

37. H.N. Han, C.G. Lee, C.S. Oh, T.H. Lee, and S.J. Kim: Acta Mater., 2004, vol. 52, pp. 5203-14.

38. P. Jacques, Q. Furnémont, A. Mertens, and F. Delannay: Philos. Mag. A, 2001, vol. 81, pp. 1789-1812.

39. R. Petrov, L. Kestens, A. Wasilkowska, and Y. Houbaert: Mater. Sci. Eng. A, 2007, vol. 447, pp. 285-97.

40. Y.F. Shen, L.N. Qiu, X. Sun, L. Zuo, P.K. Liaw, and D. Raabe: Mater. Sci. Eng. A, 2015, vol. 636, pp. 551-64. 